\title{
Feasibility of Using 2,3,3,3-Tetrafluoropropene (R1234yf) as a Solvent for Solid-Liquid Extraction of Biopharmaceuticals
}

\author{
John Suberu, ${ }^{\dagger}$ Peyman Yamin, ${ }^{\dagger}$ Robert Cornell, ${ }^{\dagger}$ Arya Sam, ${ }^{\dagger}$ and Alexei Lapkin*, ${ }^{\dagger}$ \\ ${ }^{\dagger}$ Department of Chemical Engineering and Biotechnology, Pembroke Street, University of Cambridge, Cambridge CB2 3RA, United \\ Kingdom \\ ${ }^{\ddagger}$ Department of Materials Science and Metallurgy, 27 Charles Babbage Road, University of Cambridge, Cambridge CB3 OFS, United \\ Kingdom
}

\section{Supporting Information}

ABSTRACT: Tetrafluoropropene (R1234yf) is the most likely replacement for tetrafluoroethane (R134a), a widely used refrigerant, propellant, and solvent, characterized by a very high global warming potential. In this study, solvation properties of R1234yf were studied experimentally and computationally for solubility of artemisinin, a precursor to the important biopharmaceutical API, and extraction of artemisinin from biomass. R1234yf was shown to be a poorer solvent than R134a for artemisinin. COSMO-RS calculations of solvation in R1234yf suggest that the decrease in performance is likely due to entropic effects. However, R1234yf was effectively used in solid-liquid extraction of Artemisia annua. The new solvent has shown an increased selectivity to the target metabolite artemisinin. This should allow for design of

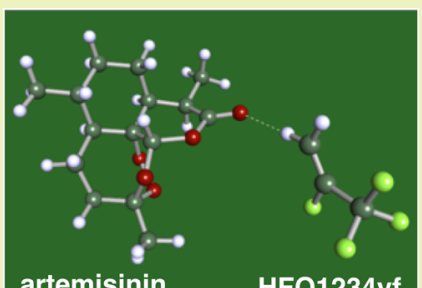

artemisinin HF01234yf more selective separation processes based on the new solvent molecule with a low global warming potential of 4 relative to $\mathrm{CO}_{2}$. KEYWORDS: R1234yf, R134a, Artemisinin, COSMO-RS, Extraction, Solubility

\section{INTRODUCTION}

Solid-liquid and liquid-liquid extraction processes are ubiquitous in industry, both as downstream purification methods and as primary methods of producing molecules of interest, e.g., in extraction of flavor, fragrance, and bioactive compounds. At the end of a process, solvents may be recovered but frequently are incinerated to produce energy. This represents a non-negligible contribution to the environmental impact of processing industries. A number of solvents, such as chlorinated solvents, are now actively discouraged from industrial use, and some companies have developed internal tools to influence the choice of solvents for process development, e.g., GSK's (GlaxoSmithKline) solvent selection guide.

One of the strategies to minimize environmental impact from solvents use and production is to encourage the use of clean solvents, such as water, supercritical carbon dioxide $\left(\mathrm{scCO}_{2}\right)$, ionic liquids, and bioderived solvents. ${ }^{2}$ Among these, solvents with high boiling points require an additional separation step since their distillation to recover the compounds of interests is not feasible, whereas $\mathrm{scCO}_{2}$, as well as other supercritical fluids (water, propane, etc.) require only a partial depressurization to recover the target compounds. This is a significant technological advantage, potentially leading to intensification of separation processes based on sc-fluids, and this feature of supercritical solvents is being widely explored not only in separations but also in design of new catalytic processes. ${ }^{3}$

Another class of solvents with low boiling points are low hydrofluorocarbons (HFCs), among which tetrafluoroethane (1) has been reported as an efficient solvent for hydrogenations $s^{4,5}$ and as a solvent for solid-liquid extraction from biomass. $^{6-9}$ Hydrofluorocarbons, such as tetrafluoroethane (R134a), are rather expensive to produce. What allowed their limited commercial applications as solvents is their large-scale use as a heat transfer medium in refrigeration and as a drug delivery system in inhalers. ${ }^{10,11}$ The overall use of R134a as a solvent is negligible in comparison to the annual use and loss to the environment of R134a as a propellant and a refrigerant. However, the Montreal and Kyoto protocols directed signatory countries to phase out the use of compounds with high ozone depleting (ODP) and global warming potentials (GWP). ${ }^{12,13}$ Consequently, the European Commission in May 2014 published a formal notice for registration and application for quota allocation to companies marketing hydrofluorocarbons (HFCs) in the European Union. This was done to limit future sale and use of HFCs to one-fifth of 2014 use level by 2030. ${ }^{14}$ Similarly, in September 2014, the United States government, with support from the manufacturing industry, announced commitment for further reduction in global HFC consumption by the equivalent of 700 million tonnes of $\mathrm{CO}_{2}$ by $2025^{15}$

In response to this accelerated phase out of HFCs, the manufacturing industry has identified hydrofluoroolefins (HFOs) as a promising "next generation" replacement for HFCs in their most common applications as thermal fluids. Special focus is on the two main HFO isomers, 2,3,3,3tetrafluoroprop-1-ene (R1234yf, 2) and trans-1,3,3,3-tetrafluoropro-1-ene (R1234ze). These substances are judged to be realistic alternatives to HFCs in industrial applications such as

Received: December 16, 2015

Revised: February 26, 2016

Published: March 17, 2016 
cooling media. ${ }^{16}$ Both isomers, R1234yf and R1234ze, have low GWPs, with atmospheric lifetimes of about 10.5 and 16.4 days, respectively. $^{17}$

R1234yf (2) is a hydrofluoroolefin containing four fluorine atoms (Scheme 1) with similar thermodynamic properties as

Scheme 1. Structures of Molecules of Interest to This Study: 2,3,3,3-Tetrafluoropropene (R1234yf), Tetrafluoroethane (R134a), and Metabolites of A. annua

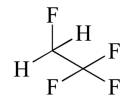

R134a (1)

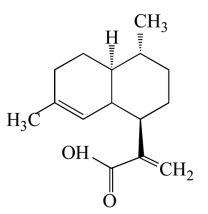

dihydroartemisinic acid (4)<smiles>C=C(F)C(F)(F)F</smiles>

R1234yf(2)<smiles>C=C1C(=O)O[C@]23[C@H]1CC[C@H](C)[C@H]2CC[C@]1(C)O[C@@H]13</smiles>

arteannuin B (5)

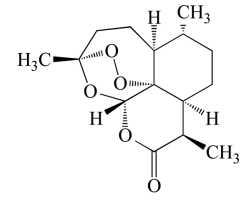

$\operatorname{artemisinin}(\mathbf{3})$
Table 1. Physicochemical Properties of R134a and R1234yf

\begin{tabular}{llll}
\multicolumn{1}{c}{ properties } & \multicolumn{1}{c}{ R134a } & R1234yf & \multicolumn{1}{c}{ ref } \\
boiling point $T_{\mathrm{b}}(\mathrm{K})$ & 247.15 & 243.75 & 20,21 \\
critical point $T_{\mathrm{c}}(\mathrm{K})$ & 374 & 367.7 & $22-24$ \\
& 375.15 & 368.15 & 19 \\
vapor pressure at $293 \mathrm{~K}(\mathrm{kPa})$ & 572 & 593.4 & 20,25 \\
molar mass $\left(\mathrm{kg} \mathrm{kmol}^{-1}\right)$ & 102.3 & 114.04 & \\
vapor density at $298.15 \mathrm{~K}\left(\mathrm{~kg} \mathrm{~m}^{-3}\right)$ & 37.94 & 32.34 & 22 \\
critical pressure $(\mathrm{MPa})$ & 4.0593 & 43.382 & 19 \\
GWP & 1430 & 4 & 26 \\
atmospheric life time & 14 years & 11 days & 27 \\
\hline
\end{tabular}

R134a, shown for comparison in Table 1. The short atmospheric lifespan of hydrofluoroolefins is a consequence of the relative instability in the atmospheric environment of the $\mathrm{C}=\mathrm{C}$ double bond. As a result, the potential for instability of these fluids is a concern in some applications, where these are being considered as a "drop-in" replacement for the more stable HFCs. ${ }^{18,19}$

Consequently, the stability and phase behavior of R1234yf in different fluid systems and at a broad range of conditions are currently being keenly investigated, especially in the automotive and refrigeration industries. ${ }^{28}$ For applications in the refrigeration industry, Nato et al. studying the phase equilibria of oil-refrigerant systems found that R1234yf was more stable in polyolester (POE) lubricant oils than 1,1,1,2-tetrafluoroethane (R134a). However, R1234yf offers lower absorption rates in the oil than what was observed for R134a. ${ }^{29}$ Likewise, Bobbo et al. investigated the solubility of R1234yf and R134a in commercial polyalkylene glycol (PAG) lubricants at isothermal conditions for similar applications and found problematic phase separation behavior for the R1234yf-PAG system at temperatures above $293.15 \mathrm{~K}^{30}$
To our knowledge, there is no literature investigating the suitability of R1234yf as a greener replacement fluid for hydrofluoroalkanes as an extraction solvent. Therefore, here we investigated experimentally and through computational modeling the solvation properties of R1234yf using artemisinin and its extraction from Artemisia annua as an example of a currently relevant industrial biopharmaceutical product.

\section{EXPERIMENTAL SECTION}

Chemicals. Artemisinin used for the solubility experiments was donated by Ipca Laboratories Ltd. (India) and Botanical Extracts EPZ Ltd. (Kenya). The artemisinin (>99.0 wt \%) used for HPLC and LCMS standard calibration was obtained from Sigma-Aldrich (U.K.) along with HPLC grade acetonitrile and LC-MS grade formic acid in water and in acetonitrile. 2,3,3,3-Tetrafluoroprop-1-ene (R1234yf) was sourced from A-gas (Bristol, U.K.) with the manufacturer specifications given in Table 2 .

Table 2. R1234yf Specifications According to the Manufacturer

\begin{tabular}{lcl}
\multicolumn{1}{c}{ parameters } & specification & \multicolumn{1}{c}{ units } \\
purity & $\geq 99.50$ & $\%$ wt liquid phase \\
water & $\leq 10.00$ & $\mathrm{ppm}$ wt liquid phase \\
acidity & $\leq 1.00$ & $\mathrm{ppm}$ wt liquid phase \\
residue & $\leq 0.01$ & $\% \mathrm{v} / \mathrm{v}$ liquid phase \\
noncondensable & $\leq 1.50$ & $\% \mathrm{v} / \mathrm{v}$ vapor phase at $298.15 \mathrm{~K}$
\end{tabular}

Solubility. Experimental Setup. The setup included two pressurized $35 \mathrm{~mL}$ tube vessels (Q-tube, Sigma-Aldrich) as shown in Figure 1. The vessels were connected by PTFE tubing (OD 3.175

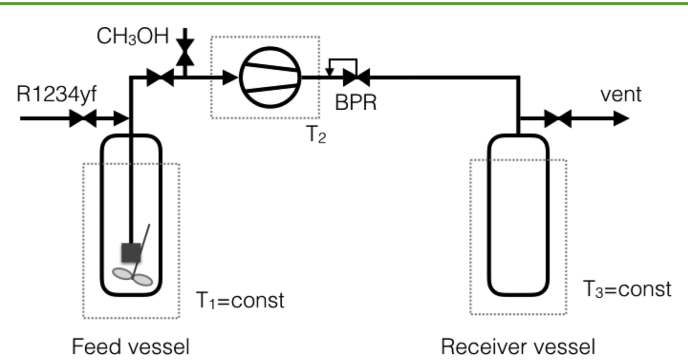

Figure 1. Schematic diagramme of the solubility measurements setup.

mm) via an HPLC pump (Knauer) of a Vaportec R2 pumping unit (U.K.) and a backpressure regulator (BPR, $87 \mathrm{psi}$ ). The aluminum headers of the Q-tube vessels were modified, each fitted with a medium-pressure metering valve (Swagelok U.K.), and feed vessel was equipped with a pressure gauge. Samples and solvent were withdrawn from the feed vessel via an immersed sample tube $(120 \mathrm{~mm})$ with a custom built $6 \mathrm{~mm}$ OD, $7 \mathrm{~mm}$ length filter end-cup to hold filters (Green Combi, Swan). The feed vessel was thermostated to the required temperature $\left(T_{1}\right)$ using a water-recirculating bath (K20 ThemoScientific, U.K.). The pump head was cooled with an ice pack $\left(T_{2}\right)$.

The receiver vessel was kept cool in an icebox $\left(T_{3}\right)$. An R1234yf (or R134a) cylinder or methanol-containing vessel was connected to the feed vessel via a shut-off valve.

Batch Solubility Measurements with Solvent Removal by Pumping. Excess artemisinin $(\sim 600 \mathrm{mg})$ was weighed and placed in the feed vessel along with a magnetic bar. The vessel and its content were weighed before and after adding about $20 \mathrm{~mL}$ of R1234yf (or R134a) from a pressurized cylinder. The solvent level in the vessel was marked. The vessel and content were sonicated in a thermostated sonication bath for $30 \mathrm{~min}$ at $\sim 5$ degrees above the target solubility temperature (283, 293, 303, and $313 \mathrm{~K})$. Temperature during sonication was kept relatively constant by stirring into the bath 
small pieces of ice when necessary. After sonication, the vessel was transferred to the water bath and thermostated to the target temperature, and the solution was left to equilibrate over $2 \mathrm{~h}$ under gentle stirring. Thereafter, stirring was stopped, and the system was allowed to equilibrate for further $30 \mathrm{~min}$. The metering valve on the feed vessel was open, and about $10-12 \mathrm{~mL}$ of solution was pumped into the receiver vessel. The new solution level in the feed vessel was marked. The feed vessel was disconnected from the system and weighed. The receiving vessel was slowly depressurized allowing gentle evaporation and venting of all R1234yf (or R134a). Since the saturated solution was pumped between the vessels, all wetted internal surfaces would be covered with the solute precipitate. Methanol $(\sim 10 \mathrm{~mL})$ was used to wash the connecting line and pump head from the precipitated solute into the receiving vessel. The volume of the methanol solution in the receiver vessel was also determined. An aliquot of the methanol solution's filtrate was diluted appropriately for HPLC and LCMS analysis.

The amount of artemisinin in the sample was determined by recalculating the peak area obtained from HPLC or MS chromatograms to the corresponding concentration using an external standard multilayer calibration curve method and calculated according to eq 1 .

$$
\text { Amount (of sample) }=\text { Response } \times \operatorname{RF} \times M \times D
$$

where "Response" is the peak area in a chromatogram, RF is the response factor determined from a calibration curve, $M$ is the multiplier to account for sample weight and standard purity factor, and $D$ is the dilution factor used in the sample preparation. The amount of solvent (R1234yf/R134a) and solute (artemisinin) were thereafter expressed as moles and used to calculate the corresponding mole fractions.

"Shake Flask" Method for Validation of the Solvent Removal Method. The "shake flask" method described by Higuchi and Connors $^{31}$ is the most reliable and widely used experimental drug solubility measurement method, even though it is relatively time consuming. ${ }^{32,33}$ This method was used to validate the method described above for the pressurized solvents. Because the "shake flask" method is not suitable for pressurized liquids, we used methanol as an alternative solvent for the validation tests. Briefly, an excess amount ( $150 \mathrm{mg}$ ) of artemisinin was added to $7 \mathrm{~mL}$ of anhydrous methanol $(99.8 \%)$ in a $10 \mathrm{~mL}$ glass vial containing a magnetic stirrer. The sealed vial was placed in a thermo-stated water bath kept at $295 \mathrm{~K}$ and gently stirred at $\sim 150 \mathrm{rpm}$ for $25 \mathrm{~h}$ to achieve a saturated solution in equilibrium with the solid phase. Thereafter, stirring was stopped, and the content of the vial was allowed to phase separate over $2 \mathrm{~h}$. An aliquot of the supernatant was passed through a syringe filter $(0.2 \mu \mathrm{m}$, Chromacol, Thermo Scientific, U.K.) prewetted with the saturated solution and collected in an HPLC vial for analysis. The corresponding flow method solubility measurements were conducted like for R1234yf above but with methanol as the solvent and without the final methanol wash. An aliquot was collected in an HPLC vial for analysis.

Biomass Extraction Using Liquid and Pressurized Solvents. Here, $0.5 \mathrm{~g}$ of dried and ground Artemisia annua plant leaves were extracted with $\sim 20 \mathrm{~mL}$ of either ethyl acetate, hexane modified with $5 \%$ ethyl acetate, or R1234yf in a comparative trial. The exhaustive extraction with ethyl acetate was performed based on the literature method. ${ }^{6}$ All extractions with liquid solvents were carried out in $50 \mathrm{~mL}$ conical flasks. In the case of R1234yf, extraction was performed in a pressure Q-tube (feed vessel in Figure 1). In the case of all solvents, the biomass solvent-containing vessels were sonicated for $30 \mathrm{~min}$ in a thermo-stated bath at $295 \mathrm{~K}$.

In the case of conventional solvents, the extracts were filtered through a $1.0 \mu \mathrm{m}$ (Whatman filter) paper to give the crude extract. One milliliter of extract was air-dried in a fume hood and reconstituted in $1 \mathrm{~mL}$ of acetonitrile, which was diluted appropriately for both HPLC and LC-MS analyses.

For R1234yf, the Q-tube was filled with biomass, then solvent, and extracted as described above. After sonication the Q-tube was connected to the setup shown in Figure 1 as the feed vessel. The crude extract was filtered and pumped into the second Q-tube (receiver vessel) as described above. The receiver vessel was carefully depressurized to gently remove the solvent. The solvent-free extract left in the tube was reconstituted in an appropriate solvent and dilutions for analysis.

HPLC Analysis Method. The HPLC instrument (Shimadzu Prominence) was equipped with a degassing unit (DGU-20A), a binary pumping module (LC-20AD), an autosampler (SIL-20A), a communication module (CBM-20A), a diode array detector module (SPD-M20A), and an oven (CTO-20AC). The method by Lapkin et al. $^{34}$ was used in the analysis, with modifications. Briefly, a mobile phase mixture of acetonitrile and water $(60: 40)$ was used in an isocratic, $15 \mathrm{~min}$ run on a C18 $(2.7 \mu \mathrm{m}, 4.6 \mathrm{~mm} \times 150 \mathrm{~mm})$ Cortecs column (Waters Ltd., U.S.A.), which was thermo-stated at $303 \mathrm{~K}$. Chromatographic detection was at $210 \mathrm{~nm}$ wavelength with a $10 \mu \mathrm{L}$ injection volume.

Liquid Chromatography Method for LC-MS. A Shimadzu Prominence HPLC system coupled to a Xevo tandem quadrupole mass spectrometer (Waters Corp., Milford, MA, U.S.A.) was used for the analysis of artemisinins based on the method by Suberu et al. ${ }^{35}$ Briefly, a Cortecs C18 column $(100 \mathrm{~mm} \times 2.1 \mathrm{~mm}, 2.7 \mu \mathrm{m})$ (Waters Corp., U.S.A.) was used for separation of the metabolites and was set to $303 \mathrm{~K}$. The mobile phase consisted of A ( $0.1 \%$ formic acid in water) and $\mathrm{B}$ ( $0.1 \%$ formic acid in acetonitrile). Chromatographic separation was achieved using a linear gradient: $0-7.0 \mathrm{~min}, 25-98 \% \mathrm{~B} ; 7-9.5$ min, $98 \%$ B; $9.5-10 \mathrm{~min}, 98-25 \% \mathrm{~B} ; 10-15 \mathrm{~min}, 25 \% \mathrm{~B}$; at a flow rate of $0.4 \mathrm{~mL} \mathrm{~min}^{-1}$.

Multiple Reaction Monitoring (MRM) Method. The tandem MS experiments were carried out in positive electrospray ionization (ESI + ), and acquisition was performed in MRM mode according to Suberu et al. ${ }^{36}$ The cone and desolvation gas flow rates were set at 45 and 800 $\mathrm{L} \mathrm{h}^{-1}$, respectively, while the capillary voltage and source and and desolvation temperatures were similar for all analytes at $40 \mathrm{kV}$ and 423 and $623 \mathrm{~K}$, respectively. Data were acquired by MassLynx (v. 4.1) and processed for quantification with QuanLynx (v. 4.1, Waters Corp., Milford, MA, U.S.A.).

DSC Enthalpy of Fusion Determination. The enthalpy of fusion was determined for both the solute (artemisinin) and the solvent (R1234yf) using a Q2000 differential scanning calorimeter (TA Instruments, U.K.). The Q2000 instrument was calibrated using indium $\left(T_{\mathrm{m}}=429.75 \mathrm{~K}\right.$ and $\left.\Delta H_{\text {fusion }}=27.81 \mathrm{~J} \mathrm{~g}^{-1}\right)$. A quantity of artemisinin was weighed by difference into an aluminum pan, and the heat of fusion determination was carried out over a temperature range from 273 to $473 \mathrm{~K}$. The solvent (R1234yf) was also measured in the range from 113 to $273 \mathrm{~K}$ with a heating rate of $10 \mathrm{~K} \mathrm{~min}^{-1}$ and

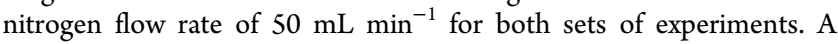
sample of R1234yf was cooled by dispensing an aliquot from a pressurized vessel into an empty beaker, which was kept in a bath of liquid nitrogen. A small liquid sample was scoped into an aluminum pan, which was preweighed and cooled with liquid nitrogen. The pan was sealed and reweighed before placing into the DSC instrument for enthalpy of fusion measurement. The DSC data were analyzed using the Universal Analysis 2000 software.

Computational Methods. COSMO-RS ${ }^{37}$ was used for computational modeling of the solubility. COSMO-RS allows for the calculation of the chemical potential of liquid mixtures ${ }^{38}$ and can be used for predicting phase equilibria and liquid state thermodynamics. COSMOtherm (v. 15.0.0, COSMOlogic GmbH \& Co. KG) was used for all COSMO-RS calculations. The starting molecular conformations were obtained using COSMOconf (v. 3.0, COSMOlogic GmbH \& Co. $\mathrm{KG}$ ) with the BP-TZVP parametrization. In COSMO-RS, the solubility of solute $j$ in solvent $i$ is calculated according to eq 2 .

$$
\log _{10} X_{j, \text { sol }}=\mu_{j, \mathrm{P}}-\mu_{j, i}-\max \left(0, \Delta G_{\mathrm{fus}}\right) /(R T \ln 10)
$$

where $X_{j, s o l}$ denotes the solubility in mole fractions, and $\mu_{j, \mathrm{P}}$ and $\mu_{j, i}$ are chemical potentials of all compounds $j$ in their pure form and in the mixture with $i$. The free energy of fusion $\left(\Delta G_{\text {fus }}\right)$ is needed for the calculation of absolute solubility in the case of a solid solute. In the case of a temperature-dependent free energy of fusion, the heat capacity of fusion $\left(\Delta C_{\mathrm{P}, \text { fus }}\right)$ and hence $\Delta G_{\text {fus }}$ are approximated by as given in eq 3. 


$$
\begin{aligned}
\Delta C_{\mathrm{P}, \text { fus }} \approx & \Delta S_{\text {fus }}=\Delta H_{\text {fus }} T_{\mathrm{m}} \\
\Delta G_{\text {fus }}(T)= & \Delta H_{\text {fus }}\left(1-\frac{T}{T_{\mathrm{m}}}\right)-\Delta C_{\mathrm{P}, \text { fus }}\left(T_{\mathrm{m}}-T\right) \\
& +\Delta C_{\mathrm{P}, \text { fus }} T \ln \frac{T_{m}}{T}
\end{aligned}
$$

For a qualitative comparison between solvents, the activity coefficient at infinite dilution has been calculated and used. This quantity is obtained directly from the chemical potentials calculated using COSMO-RS as it is directly related to the chemical potential by eq 4 .

$$
\mu_{j}=\mu_{j}^{0}+R T \ln a_{j}
$$

where $a$ is the activity of solute $j$ and is given as $a_{j}=X_{j} \gamma_{j}$, with $\gamma_{j}$ being the activity coefficient of solute $j$ and $x_{j}$ its mole fraction. From the condition of equilibrium between the two solvents, we know that the chemical potential and thus the activity of the solute should be equal in both solvents. Hence, $X_{S_{1}} \gamma_{S_{1}}=X_{S_{2}} \gamma_{S_{2}}$, where $S_{1}$ and $S_{2}$ denote the two solvents being compared. This relation makes it possible to compare the activity coefficient of a solute in two different solvents and draw conclusions on its mole fraction in them at the solubility limit.

In COSMO-RS, each molecule is situated in a cavity surrounded by an ideal conductor, its surface is divided into segments, and the electronic charge on it is calculated using quantum mechanics. Every segment is characterized by its area and the density of the so-called screening charge on its surface. The screening of the charge on the surface is complete at the presence of the ideal conductor surrounding. In COSMO-RS, every molecule is then treated as an ensemble of interacting surface segments. The distribution of the charge density among segments is a fundamental property of each molecule in COSMO-RS and is called the profile of that molecule. The calculation of the chemical potential in COSMO-RS is then done by statistically taking into account all possible contacts between all the segments present in a liquid mixture and involves various interaction energies, such as electrostatic, hydrogen bonding, and van der Waals.

\section{RESULTS AND DISCUSSION}

Validation of Solvent Flow Solubility Measurements. The experimental setup for the solvent flow method was validated against a "shake flask" method. The use of a cellulose filter in the feed vessel Q-tube limits the range of suitable solvents. Acetonitrile and acetone were found to degrade the cellulose-based filter. However, the filter was stable in methanol, ethanol, R134a, and R1234yf in our investigation.

Table 3 shows the mole fraction solubility of artemisinin in anhydrous methanol at $295 \mathrm{~K}$. This corresponds to $8.71 \pm 0.03$

Table 3. Solubility of Artemisinin (mole fraction $\times 10^{3}$ ) in Anhydrous Methanol at $295 \mathrm{~K}$

\begin{tabular}{|cccc|}
\hline method & replicate 1 & replicate 2 & replicate 3 \\
\hline shake flask & $1.237 \pm 0.008$ & $1.248 \pm 0.004$ & $1.248 \pm 0.002$ \\
solvent flow & $1.236 \pm 0.004$ & $1.254 \pm 0.005$ & $1.248 \pm 0.002$ \\
\hline
\end{tabular}

and $8.70 \pm 0.02 \mathrm{mg} \mathrm{mL}^{-1}$ obtained for the flow vessel and the "shake flask" methods, respectively. The result also compares favorably to the $8.17 \mathrm{mg} \mathrm{mL}^{-1}$ (or mole fraction $1.17 \pm 0.02 \times$ $10^{3}$ ) value obtained by Nti-Gyabaah et al. ${ }^{39}$ for artemisinin solubility in pure methanol at a similar temperature, thereby validating the solvent flow method of solubility determination.

Experimental Determination of Solubility of Artemisinin in R1234yf and R134a. The experimental solubility of artemisinin in R1234yf was determined at various temperatures as shown in Figure 2 alongside the solubility in R134a at $293 \mathrm{~K}$, shown in Table 4. The trend observed for artemisinin solubility in R1234yf shows an increase with an increase in temperature over the range investigated. In terms of solubility comparison between the two solvents, the solubility of artemisinin in R134a was found to be about 7 times higher that in R1234yf at $293 \mathrm{~K}$.

Comparatively, at low temperatures $(283.15 \pm 1.00 \mathrm{~K})$, the solubility of artemisinin in R1234yf $(1.44 \pm 0.02)$ was superior to its solubility (mole fraction $\left.\times 10^{3}\right)$ in methanol $(0.56 \pm$ $0.01)$, acetonitrile $(0.84 \pm 0.01)$, butanol $(0.85 \pm 0.02)$, hexane $(0.05 \pm 0.01)$, and heptane $(0.05 \pm 0.01)$ reported in the literature. ${ }^{39}$ Even at room temperature $(294.15 \pm 1.00 \mathrm{~K})$, the solubility of artemisinin in R1234yf $(1.53 \pm 0.08)$ is comparable to that of acetonitrile $(1.57 \pm 0.04)$ and better than those for methanol $(1.17 \pm 0.02)$, hexane $(0.11 \pm 0.02)$, and heptane $(0.13 \pm 0.02)$. This would suggest that R1234yf is a better solvent for artemisinin at room and lower temperatures than some conventional solvents.

Determination of Enthalpy of Fusion and Melting Point. The melting point and enthalpy of fusion for artemisinin crystals and R1234yf used in our experiments are shown in Table 5. The two peaks in the DSC thermogram correspond to the crystalline and amorphous forms of artemisinin. Although a range of melting temperatures (426.15-432.15 K) have been reported in the literature for artemisinin, ${ }^{39-41}$ the melting temperature $\left(T_{\mathrm{m}}\right)$ obtained in our determination for the crystalline state $(424.96 \pm 0.3 \mathrm{~K})$ is similar to that reported for the orthorhombic polymorph $(424.55)$ by Horosanskaia et al., ${ }^{40}$ while the melting enthalpy $\left(\Delta H_{\text {fus }}\right)$ here reported $\left(24,545.94 \pm 250.5 \mathrm{~J} \mathrm{~mol}^{-1}\right)$ corresponds closely to values $\left(24,300 \pm 400 \mathrm{~J} \mathrm{~mol}^{-1}\right)$ obtained by Nti-Gyabaah et al. ${ }^{39}$ for artemisinin. Differences in the analytic conditions, e.g., the heating rate employed in these DSC experiments, is suggested as a possible cause for the variation in the reported $T_{\mathrm{m}}$ and $\Delta H_{\text {fus }}$ for the compound. ${ }^{40}$ In a previous study, ${ }^{6}$ the value for the free energy of fusion of artemisinin at $294 \mathrm{~K}$ was estimated to be 11.39 and $8.9 \mathrm{~kJ} \mathrm{~mol}^{-1}$, using a fitting of COSMO-RS calculations to the solubility data for ethanol and ethyl acetate, respectively. In this work, using the experimentally measured fusion enthalpy and temperature, the free energy of fusion at $294 \mathrm{~K}$ calculated using eq 3 is $6.28 \mathrm{~kJ} \mathrm{~mol}^{-1}$. We have hence used the values obtained from our experimentally measured fusion enthalpy and temperature for the calculation of the free energy of fusion and subsequently the absolute values for the solubility of artemisinin in R1234yf at different temperatures given in Table 6.

To our knowledge, there are no literature values for the melting temperature and the heat of fusion of R1234yf. The obtained experimental values, Table 5, were used in COSMORS calculation of chemical potential of the solid solute in a liquid mixture.

Computational Modeling of Artemisinin Solubility in R1234yf and Other Solvents. Figure 3A-F shows the most probable configuration of artemisinin and six solvent molecules. The configurations were obtained using COSMOtherm based on the contact probability of molecular surface segments. Each dashed line depicts a possible hydrogen bond. It is shown that all hydrogen bond donors attach to the oxygen atom of the carbonyl carbon (Figure 3 A, D, and F). Further analysis showed that the second and third oxygens correspond to the second and third most probable hydrogen bonds (Figure 3B). Solvents with a hydrogen bonding acceptor in turn attach to hydrogens on the ring (Figure 3C).

The absolute values of the calculated solubilities in Table 6 deviate from those obtained experimentally in Table 4 but are in the expected range regarding the quantitative accuracy of 


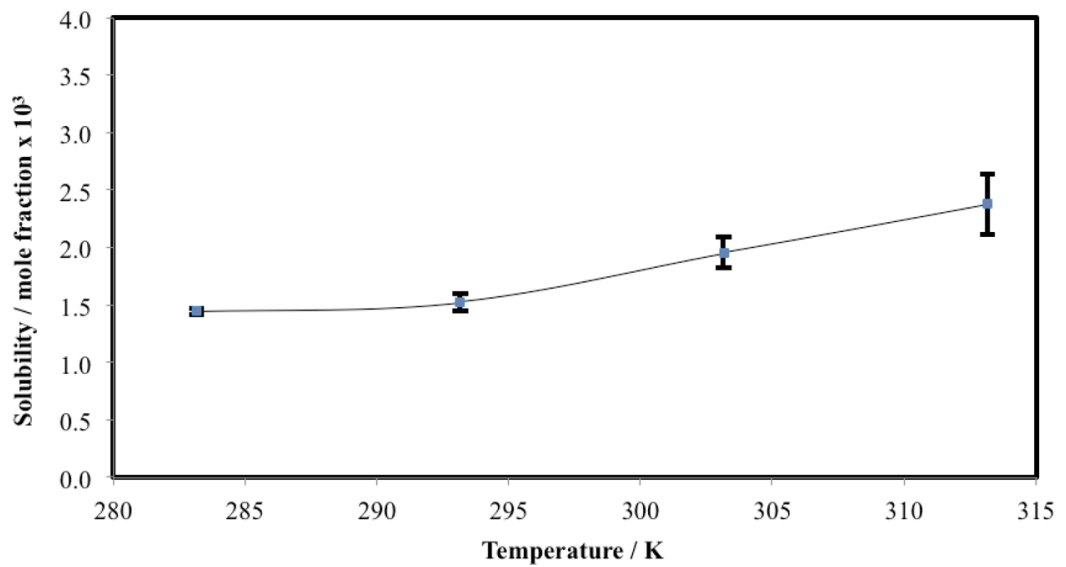

Figure 2. Experimental solubility of artemisinin in R1234yf as a function of temperature.

Table 4. Solubility of Artemisinin in R1234yf and in R134a at Given Temperatures

$\begin{array}{ccc}\text { temperature }(\mathrm{K}) & \text { pressure }(\mathrm{KPa}) & \text { solubility }\left(\text { mole fraction } \times 10^{3}\right) \\ \text { R1234yf } & & \\ 283.15 & 307.5 & 1.44 \pm 0.02 \\ 293.15 & 450.0 & 1.53 \pm 0.08 \\ 303.15 & 615.0 & 1.95 \pm 0.14 \\ 313.15 & 820.0 & 2.38 \pm 0.26 \\ \text { R134a } & & \\ 293.15 & 475.0 & 8.55 \pm 0.13\end{array}$

Table 5. Enthalpy of Fusion and Melting Points of Artemisinin and R1234yf

$\begin{array}{lcc}\text { compound } & \text { melting temperature }(\mathrm{K}) & \Delta H_{\text {fus }}\left(\mathrm{J} \mathrm{mol}^{-1}\right) \\ \text { artemisinin } & 424.96 \pm 0.3 & 24,545.94 \pm 250.5 \\ \text { R1234yf } & 120.18 \pm 0.4 & 458.28 \pm 46.0\end{array}$

Table 6. Calculated Solubility of Artemisinin in R1234yf

\begin{tabular}{cccc} 
temperature $(\mathrm{K})$ & $\log 10(x)$ & mole fraction $\left(\times 10^{2}\right)$ & concentration $\left(\mathrm{g} \mathrm{L}^{-1}\right)$ \\
283.15 & -1.296 & 5.100 & 137.891 \\
293.15 & -1.210 & 6.200 & 168.104 \\
303.15 & -1.120 & 7.600 & 206.69 \\
313.15 & -1.027 & 9.400 & 256.050 \\
\hline
\end{tabular}

COSMO-RS in the prediction of solubility values for solid solutes. ${ }^{42-44}$ Qualitatively, there is a good agreement in the trend of solubility's temperature dependence between the experimental and computationally predicted results.

In order to take a step toward understanding the solubility mechanism of R1234yf, individual contributions to the COSMO-RS chemical potential are given and compared with various other solvents in Figure 4. As is known from elementary thermodynamics, a larger activity coefficient implies a higher relative solubility. Three different energetic contributions depicted in Figure 4 are the misfit energy resulting from the electrostatic interaction due to misfit charges ${ }^{45}$ that remain due to deviation of the solvent from an ideal conductor, hydrogen bonding, and van der Waals contributions. Also, the total enthalpies, i.e., the approximate sum of the mentioned three energetic contributions and the free energy of mixing including the mixing entropy, are given.

The solvents are listed from the highest to the lowest solubility from left to right. Comparing R134a with R1234yf, it can be seen from the data shown in Figure 4 and the data in the Supporting Information that the sum totals of the energetic contributions are almost the same. The entropic contribution as found in the chemical potential, however, is the only different contribution between the two solvents. Considering this, it can be deduced that the reason why R134a is a better solvent for artemisinin than R1234yf (Table 4) is of an entropic nature. One explanation can be made on the basis of the larger chain length in R1234yf than in R134a, the fact that both have the same number of fluorine atoms, and that both can form dimers in the liquid phase. Formation of dimers in the liquid phase has been shown for R134a using Monte Carlo simulations and should, in principle, hold as well for R1234yf. ${ }^{46}$ The results shown in Figure 4 can be interpreted as a higher entropy loss due to disruption of the liquid structure caused by the introduction of solute in R1234yf. In other words, R1234yf molecules have, compared to R134a, a higher tendency to form an interconnected network. Much like the hydrophobic effect, which is a result of such a network in water, the lower solubility of artemisinin in both R134a and R1234yf is also a result of such a network. This effect is then more pronounced for $\mathrm{R} 1234 \mathrm{yf}$ than R134a. Although a more comprehensive study of this effect is needed to draw clear conclusions, this could be due to a lower repulsion between the fluorine atoms during dimer formation of R1234yf due to its relatively longer chain compared to the tightly packed R134a.

From a qualitative point of view, COSMO-RS has been able to predict the difference in the solubility of artemisinin in R1234yf and R134a. Although a clear understanding of the dissolution mechanism needs a more detailed computational and experimental study of this system, an entropic difference is suggested by COSMO-RS as the reason behind the difference between the two solvents regarding artemisinin solubility.

Figure 5 shows sigma profiles ${ }^{45}$ of the two solvents R134a and R1234yf. Sigma profiles are the probability distribution diagrams of the surface screening charge as computed by COSMO-RS. It is shown that R134a has a larger peak at the negative side of the screening charge $x$-axis, corresponding to the positive charge on the surface of the molecule. This leads to stronger hydrogen bonding donor capability, corresponding to a more negative contribution of the hydrogen bonding, shown in Figure 5. The more favorable van der Waals interaction can be associated with the larger size of the nonpolar surface of R1234yf compared to that of R134a, with COSMO-RS surface areas of 118 and $105 \AA^{2}$. 


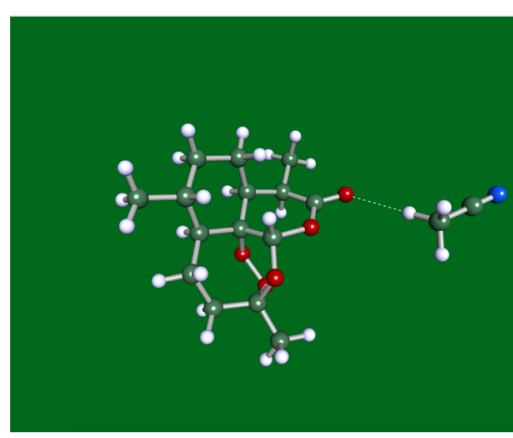

A: artemisinin-acetonitrile

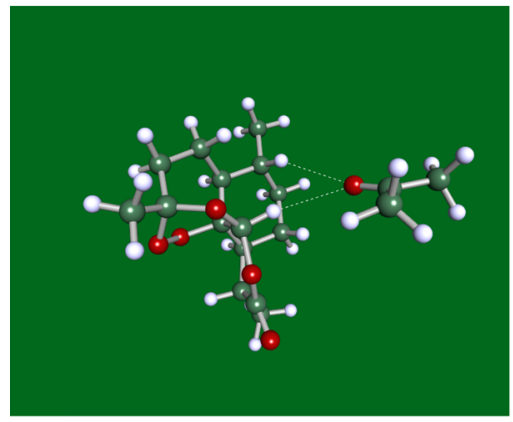

C: artemisinin-acetone

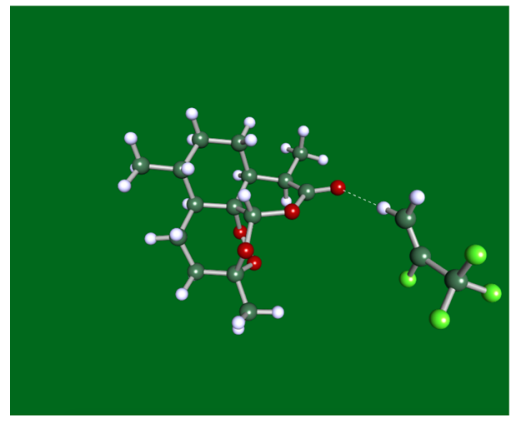

E: artemisinin-R1234yf

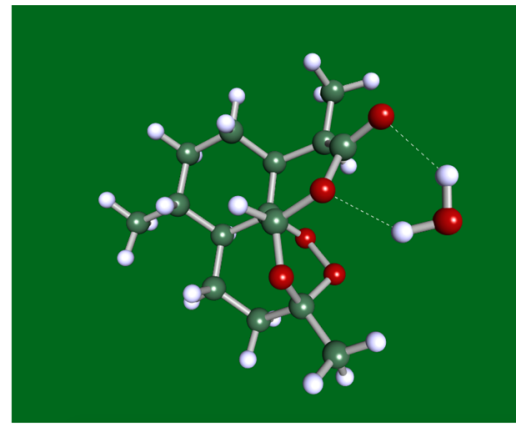

B: artemisinin-water

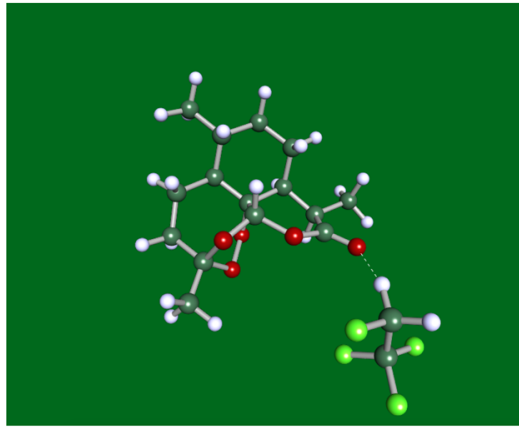

$\mathrm{D}$ : artemisinin-R134a

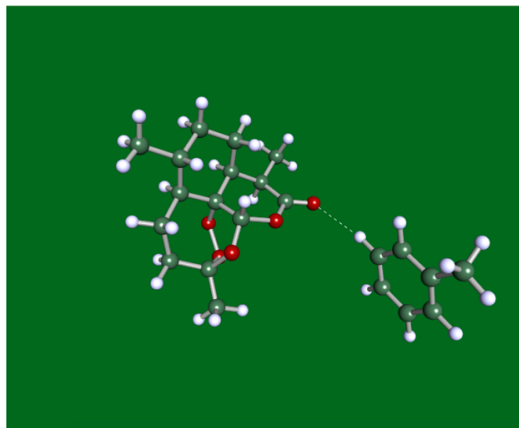

F: artemisinin-toluene

Figure 3. (A-F) Configuration of artemisinin with various solvent molecules (ball color: white, hydrogen; dark green, carbon; red, oxygen; light green, fluorine; blue, nitrogen).

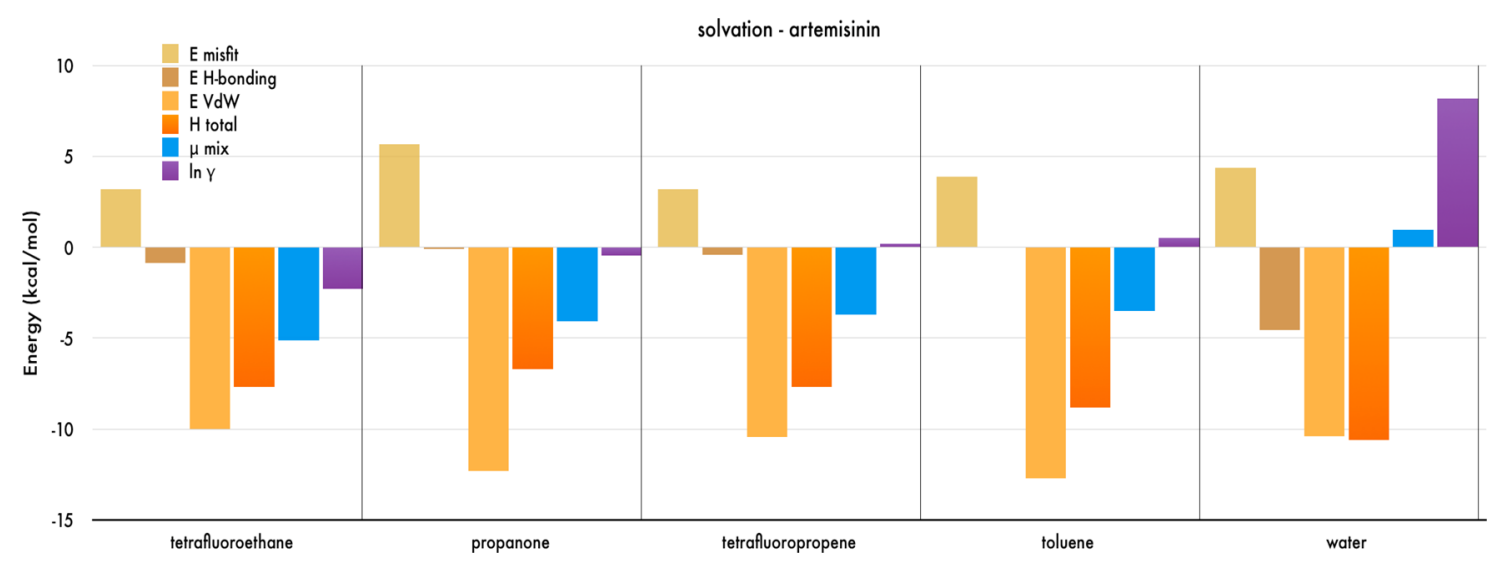

Figure 4. Contributions to the solvation chemical potential of artemisinin in common solvents calculated using COSMO-RS.

Extraction of $A$. annua with R1234yf and Comparison with Conventional solvents. A similar setup for the solubility determination was used to test extraction of $A$. annua biomass with R1234yf. This was to assess suitability of the solvent for extraction of high value compounds from the biofeedstock. The extraction efficiency for the solvent was based on exhaustive extraction of the biomass with ethyl acetate. $^{34}$ Sequential extraction over three extraction cycles 


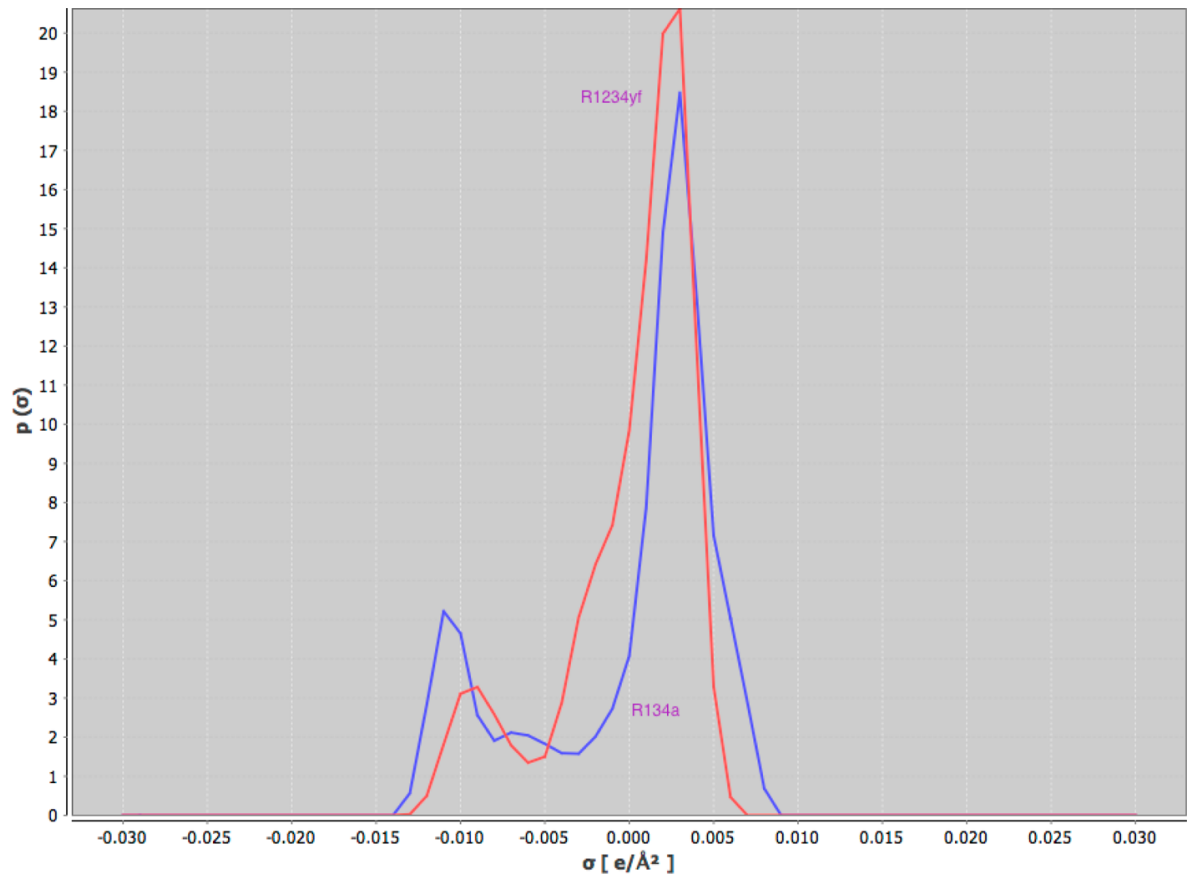

Figure 5. Sigma profiles of R134a and R1234yf.

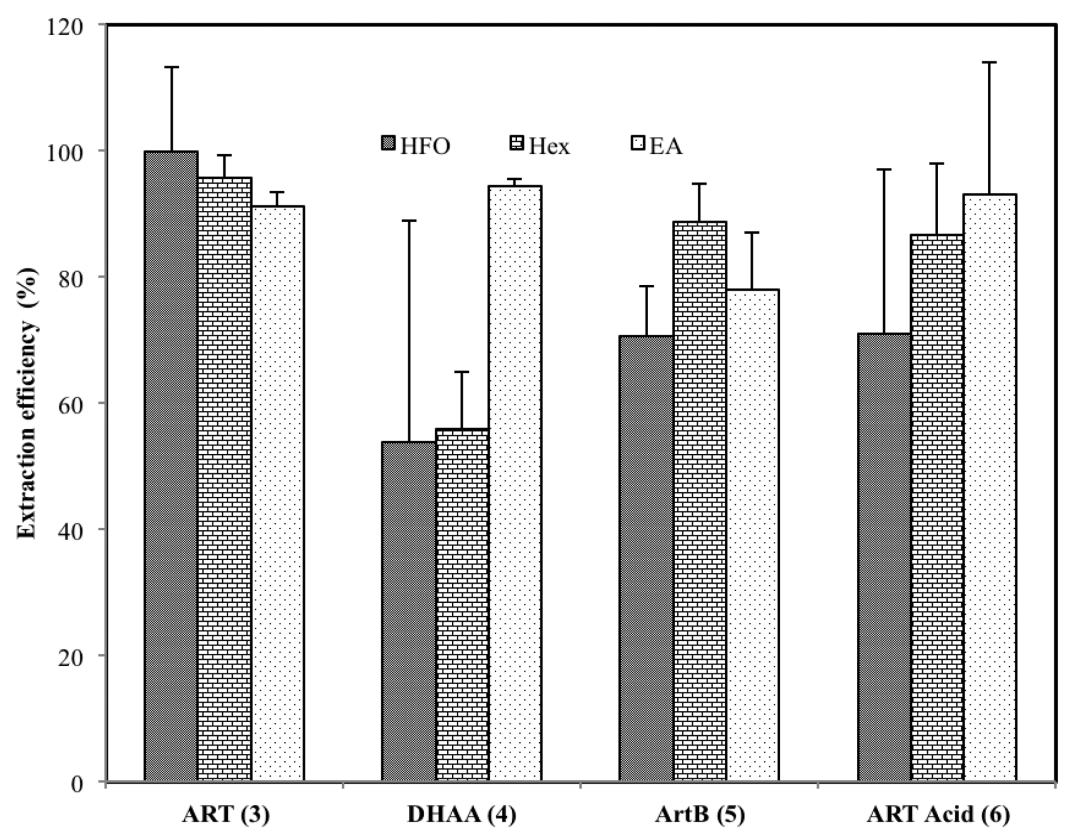

Figure 6. Efficiency of metabolite extraction with R1234yf (HFO) using method 1, hexane modified with 5\% of ethyl acetate (Hex), and pure ethyl acetate (EA).

Table 7. Comparative Analysis of Extraction of A. annua Biomass with R1234yf and Conventional Solvents

\begin{tabular}{lrrr}
\multicolumn{1}{c}{ metabolites } & \multicolumn{1}{c}{ R1234yf } & hexane with 5\% EA & ethyl acetate (EA) \\
artemisinin $\left(\mathrm{mg} \mathrm{g}^{-1}\right)$ & $12.28 \pm 1.86$ & $11.76 \pm 0.44$ & $11.2 \pm 0.26$ \\
dihydroartemisinin $\left(\mathrm{mg} \mathrm{g}^{-1}\right)$ & $1.40 \pm 0.51$ & $1.45 \pm 0.13$ & $2.46 \pm 0.03$ \\
artemisinic acid $\left(\mathrm{mg} \mathrm{g}^{-1}\right)$ & $0.25 \pm 0.06$ & $0.30 \pm 0.03$ & $0.32 \pm 0.07$ \\
arteannuin B $\left(\mu \mathrm{g} \mathrm{g}^{-1}\right)$ & $60.52 \pm 4.82$ & $76.13 \pm 4.53$ & $66.92 \pm 6.00$
\end{tabular}

gives an estimate for the amount of a target metabolite in the plant against which the extraction power of solvents for each metabolite was benchmarked to determine the efficiency of extraction shown in Figure 6.
Comparatively, R1234yf demonstrated the best extraction efficiency for artemisinin $(99.81 \pm 13.48 \%)$ of all the solvents evaluated with almost a complete extraction of the metabolite. R1234yf performed better for the extraction of artemisinin than 


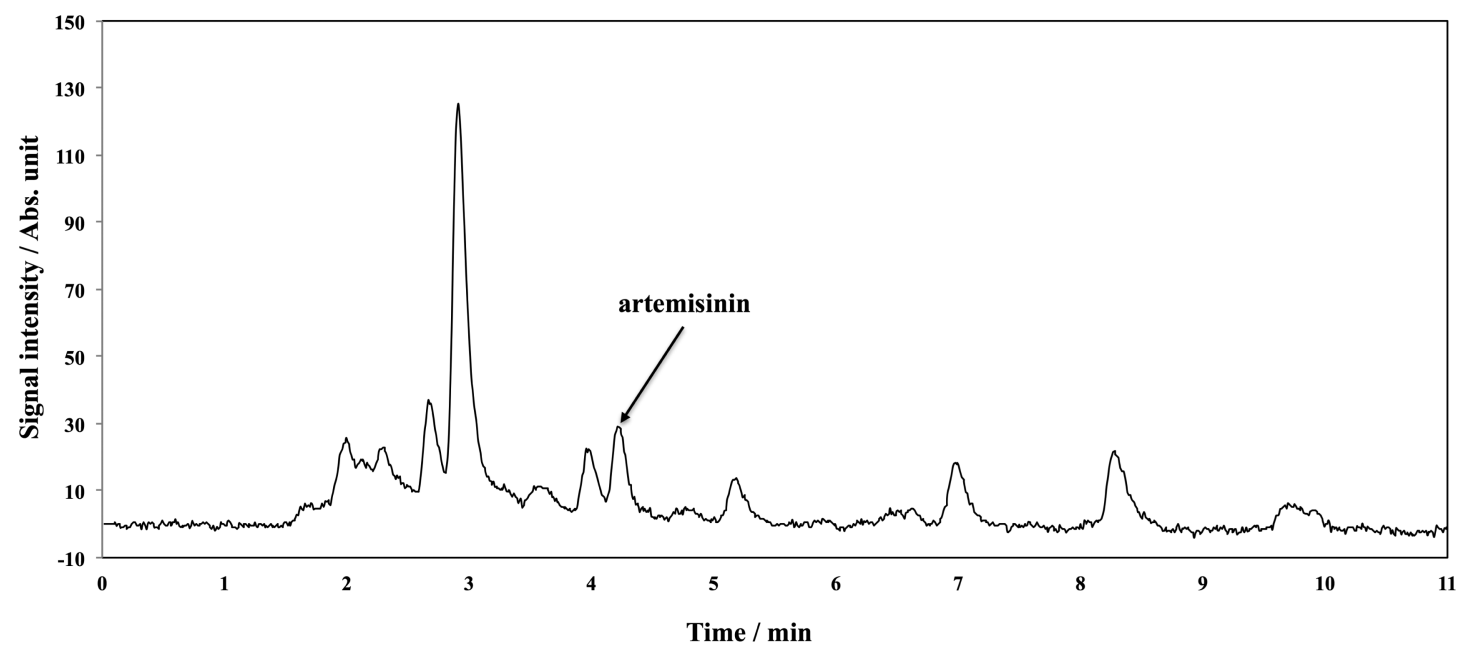

Figure 7. HPLC chromatogram of A. annua primary extract using R1234yf as extraction solvent.

a single extraction cycle with ethyl acetate $(91.06 \pm 2.35 \%)$ and the modified hexane $(95.61 \pm 3.74 \%$, Table 7$)$.

R1234yf performed poorer relative to the other solvents evaluated for the extraction of dihydroartemisinic acid, arteannuin B, and artemisinic acid. Ethyl acetate extracted above $80 \%$ of all artemisinin-related metabolites evaluated. For the extraction of DHAA, R1234yf and modified hexane performed moderately at less than $60 \%$ efficiency. Consequently, the obtained results show that if the extraction of artemisinin is the main goal, R1234yf is the solvent of choice among those investigated.

Artemisinin in Crude Extract. The R1234yf dried primary extract of $A$. annua biomass was analyzed by HPLC, and the results are shown in Figure 7 with the artemisinin peak identified. The artemisinin content in the primary extract was found to be $29.57 \pm 0.78 \mathrm{wt} \%$. This is the upper limit of the range reported in the literature for R134a (20-30 wt \%) and superior to the values for crude ethanol $(<10 \%)$, hexane, and toluene extracts (10-15\% each). ${ }^{9}$

Although the current investigation did not include purification of the primary extract, in an earlier work with conventional solvents, we employed Celite and activated carbon in the first step of purification of artemisinin extracts. ${ }^{34,47}$ A packed column containing these adsorbents and inserted in the flow extraction after the biomass bed is therefore an attractive and simple option of primary extract purification.

\section{CONCLUSIONS}

The replacement for thermal fluid R134a, being phased out due to its exceptionally high global warming potential, tetrafluoropropene (R1234yf, 2), was studied for the first time as a potential new process solvent. It was studied in solubility of a drug API precursor, artemisinin (3), and in solid-liquid extraction of the same molecule. The new solvent was found to be comparable or better to many conventional solvents in solubility of artemisinin, especially at low temperatures. It was also found to be more selective toward artemisinin in solidliquid extraction from biomass. The concentration of artemisinin in the R1234yf primary extract was superior to those reported for ethanol, hexane, and toluene and similar to R123a extraction. ${ }^{9}$ This is a significant differentiation of the new solvent compared to most solvents previously investigated for this process.

Computational study of the new solvent's solvation mechanism suggested that its poorer solvation compared to $\mathrm{R} 134 \mathrm{a}$ is due to entropic factors. COSMO-RS predictions are shown to meet the expected quantitative agreement with the experimentally measured solubility values and qualitatively match the solubility-temperature trend observed.

Given its excellent performance in solid-liquid extraction, its low GWP, and the earlier reported low process costs and process emissions from an extraction process based on R134a, ${ }^{6}$ which has very similar physical properties to R1234yf, the new solvent should outperform $\mathrm{scCO}_{2}$ in a number of industrially relevant extraction processes and in chemical manufacturing and thus has a potential to become a green process solvent ${ }^{48,49}$ in processes that allow full solvent recovery and recycling.

\section{ASSOCIATED CONTENT}

\section{S Supporting Information}

The Supporting Information is available free of charge on the ACS Publications website at DOI: 10.1021/acssuschemeng.5b01721.

Tabulated data for Figure 4. (PDF)

\section{AUTHOR INFORMATION}

\section{Corresponding Author}

*E-mail: aal35@cam.ac.uk. Fax: +44 1223334796.

Notes

The authors declare no competing financial interest.

\section{ACKNOWLEDGMENTS}

This work was funded under iCON feasibility projects scheme of EPSRC "Centre for Continuous Manufacturing (CMAC)", $\mathrm{EP} / \mathrm{IO} 33459 / 1$. We acknowledge past funding from Medicine for Malaria Ventures for the HPLC instrument and funding by University of Cambridge for the LCMS system. The assistance of Michal Presz, Lee Pratt, and Andy Hubbard with equipment modification is also acknowledged.

\section{REFERENCES}

(1) Henderson, R. K.; Jiménez-González, C.; Constable, D. J.; Alston, S. R.; Inglis, G. G.; Fisher, G.; Sherwood, J.; Binks, S. P.; Curzons, A. D. Expanding GSK's Solvent Selection Guide-Embedding Sustain- 
ability into Solvent Selection Starting at Medicinal Chemistry. Green Chem. 2011, 13 (4), 854-862.

(2) Anastas, P.; Eghbali, N. Green Chemistry: Principles and Practice. Chem. Soc. Rev. 2010, 39 (1), 301-312.

(3) Beckman, E. J. Supercritical and near-Critical $\mathrm{CO}_{2}$ in Green Chemical Synthesis and Processing. J. Supercrit. Fluids 2004, 28 (2), 121-191.

(4) Abbott, A. P.; Eltringham, W.; Hope, E. G.; Nicola, M. Hydrogenation in Supercritical 1, 1, 1, 2 Tetrafluoroethane (HFC 134a). Green Chem. 2005, 7 (10), 721-725.

(5) Saul, S.; Corr, S.; Micklefield, J. Biotransformations in LowBoiling Hydrofluorocarbon Solvents. Angew. Chem., Int. Ed. 2004, 43 (41), 5519-5523.

(6) Lapkin, A. A.; Peters, M.; Greiner, L.; Chemat, S.; Leonhard, K.; Liauw, M. A.; Leitner, W. Screening of New Solvents for Artemisinin Extraction Process Using Ab Initio Methodology. Green Chem. 2010, 12 (2), 241-251.

(7) Lapkin, A.; Adou, E.; Mlambo, B. N.; Chemat, S.; Suberu, J.; Collis, A. E.; Clark, A.; Barker, G. Integrating Medicinal Plants Extraction into a High-Value Biorefinery: An Example of Artemisia Annua L. C. R. Chim. 2014, 17 (3), 232-241.

(8) Lapkin, A. A.; Plucinski, P. K.; Cutler, M. Comparative Assessment of Technologies for Extraction of Artemisinin. J. Nat. Prod. 2006, 69 (11), 1653-1664.

(9) Khambay, B. Extraction and Isolation of Artemisinin with HFC134a, 2008. http://www.mmv.org/sites/default/files/uploads/docs/ artemisinin/06b HFC-134a.pdf (accessed March 2016).

(10) Steckel, H.; Müller, B. W. Metered-Dose Inhaler Formulations with Beclomethasone-17, 21-Dipropionate Using the Ozone Friendly Propellant R 134a. Eur. J. Pharm. Biopharm. 1998, 46 (1), 77-83.

(11) Butz, N.; Porte, C.; Courrier, H.; Krafft, M.; Vandamme, T. F.

Reverse Water-in-Fluorocarbon Emulsions for Use in Pressurized Metered-Dose Inhalers Containing Hydrofluoroalkane Propellants. Int. J. Pharm. 2002, 238 (1), 257-269.

(12) Handbook for the Montreal Protocol on Substances That Deplete the Ozone Layer; Ozone Secretariat, United Nations Environment Programme, 2006.

(13) Kyoto Protocol; United Nations Framework Convention on Climate Change: Kyoto, Japan, 1997.

(14) Mota-Babiloni, A.; Navarro-Esbrí, J.; Barragán-Cervera, Á.; Molés, F.; Peris, B. Analysis Based on Eu Regulation No 517/2014 of New Hfc/Hfo Mixtures as Alternatives of High Gwp Refrigerants in Refrigeration and Hvac Systems. Int. J. Refrig. 2015, 52, 21-31.

(15) Editors.. Seven Days: 19-25 September 2014. Nature 2014, $513,2$.

(16) Directive 2006/40/Ec of the European Parliament and of the Council of 17 May 2006 Relating to Emissions from Air-Conditioning Systems in Motor Vehicles and Amending Council Directive 70/156/ EEC. Official Journal of the European Union, 2006; pp 12-18.

(17) Tovar, C. M.; Blanco, M. B.; Barnes, I.; Wiesen, P.; Teruel, M. A. Gas-Phase Reactivity Study of a Series of Hydrofluoroolefins (HFOs) toward $\mathrm{OH}$ Radicals and $\mathrm{Cl}$ Atoms at Atmospheric Pressure and 298 K. Atmos. Environ. 2014, 88, 107-114.

(18) Navarro-Esbrí, J.; Mendoza-Miranda, J. M.; Mota-Babiloni, A.; Barragán-Cervera, A.; Belman-Flores, J. M. Experimental Analysis of R1234yf as a Drop-in Replacement for R134a in a Vapor Compression System. Int. J. Refrig. 2013, 36 (3), 870-880.

(19) Devecioğlu, A. G.; Oruç, V. Characteristics of Some New Generation Refrigerants with Low GWP. Energy Procedia 2015, 75, $1452-1457$.

(20) Smyth, H. D. The Influence of Formulation Variables on the Performance of Alternative Propellant-Driven Metered Dose Inhalers. Adv. Drug Delivery Rev. 2003, 55 (7), 807-828.

(21) Lindley, A. A.; Noakes, T. J. Consideration of Hydrofluoroolefins (HFOs) as Potential Candidate Medical Propellants, 2010. Mexichem Fluor. www.mexichemfluor.com (accessed March 2016).

(22) Ansari, N. A.; Yadav, B.; Kumar, J. Theoretical Exergy Analysis of HFO-1234yf and Hfo-1234ze as an Alternative Replacement of Hfc- 134a in Simple Vapour Compression Refrigeration System. IJSER 2013, 4 (8), 137-144.

(23) Richter, M.; McLinden, M. O.; Lemmon, E. W. Thermodynamic Properties of 2,3,3,3-Tetrafluoroprop-1-Ene (R1234yf): Vapor Pressure and $\mathrm{P}-\mathrm{P}-\mathrm{T}$ Measurements and an Equation of State. J. Chem. Eng. Data 2011, 56 (7), 3254-3264.

(24) Akasaka, R. New Fundamental Equations of State with a Common Functional Form for 2, 3, 3, 3-Tetrafluoropropene (R1234yf) and Trans-1, 3, 3, 3-Tetrafluoropropene (R-1234ze (E). Int. J. Thermophys. 2011, 32 (6), 1125-1147.

(25) Yang, Z.; Kou, L.; Mao, W.; Lu, J.; Zhang, W.; Lu, J. Experimental Study of Saturated Pressure Measurements for 2, 3, 3, 3Tetrafluoropropene (HFO-1234yf) and 2-Chloro-1, 1, 1, 2-Tetrafluoropropane (HCFC-244bb). J. Chem. Eng. Data 2014, 59 (1), 157160.

(26) Yang, L.; da Rocha, S. R. Understanding Solvation in the Low Global Warming Hydrofluoroolefin HFO-1234ze Propellant. J. Phys. Chem. B 2014, 118 (36), 10675-10687.

(27) Nielsen, O.; Javadi, M. S.; Sulbaek Andersen, M. S.; Hurley, M. D.; Wallington, T. J.; Singh, R. Atmospheric Chemistry of $\mathrm{CF}_{3} \mathrm{CF}=$ $\mathrm{CH}_{2}$ : Kinetics and Mechanisms of Gas-Phase Reactions with $\mathrm{Cl}$ Atoms, $\mathrm{OH}$ Radicals, and $\mathrm{O}_{3}$. Chem. Phys. Lett. 2007, 439 (1-3), 1822.

(28) Wang, X.; Sun, Y.; Gong, N. Experimental Investigations for the Phase Equilibrium of R1234yf and R1234ze (E) with Two Linear Chained Pentaerythritol Esters. J. Chem. Thermodyn. 2016, 92, 66-71.

(29) Neto, M. A. M.; França, R. M.; Barbosa, J. R. Convection-Driven Absorption of R-1234yf in Lubricating Oil. Int. J. Refrig. 2014, 44, 151-160.

(30) Bobbo, S.; Zilio, C.; Scattolini, M.; Fedele, L. R1234yf as a Substitute of R134a in Automotive Air Conditioning. Solubility Measurements in Two Commercial PAG Oils. Int. J. Refrig. 2014, 40, 302-308.

(31) Higuchi, T.; Connors, A. Phase-Solubility Techniques. Adv. Anal. Chem. Inst 1965, 4, 212-217.

(32) Bergström, C. A.; Norinder, U.; Luthman, K.; Artursson, P. Experimental and Computational Screening Models for Prediction of Aqueous Drug Solubility. Pharm. Res. 2002, 19 (2), 182-188.

(33) Jouyban, A.; Fakhree, M. A. Experimental and Computational Methods Pertaining to Drug Solubility. In Toxicity and Drug Testing; Acree, B., Ed.; InTech: Croatia, 2012.

(34) Lapkin, A. A.; Walker, A.; Sullivan, N.; Khambay, B.; Mlambo, B.; Chemat, S. Development of Hplc Analytical Protocols for Quantification of Artemisinin in Biomass and Extracts. J. Pharm. Biomed. Anal. 2009, 49 (4), 908-915.

(35) Suberu, J.; Song, L.; Slade, S.; Sullivan, N.; Barker, G.; Lapkin, A. A. A Rapid Method for the Determination of Artemisinin and Its Biosynthetic Precursors in Artemisia Annua L. Crude Extracts. J. Pharm. Biomed. Anal. 2013, 84 (0), 269-277.

(36) Suberu, J.; Gromski, P. S.; Nordon, A.; Lapkin, A. Multivariate Data Analysis and Metabolic Profiling of Artemisinin and Related Compounds in High Yielding Varieties of Artemisia Annua FieldGrown in Madagascar. J. Pharm. Biomed. Anal. 2016, 117 (522), 531.

(37) Klamt, A.; Krooshof, G. J.; Taylor, R. Cosmospace: Alternative to Conventional Activity-Coefficient Models. AIChE J. 2002, 48 (10), 2332-2349.

(38) Klamt, A.; Eckert, F.; Arlt, W. Cosmo-Rs: An Alternative to Simulation for Calculating Thermodynamic Properties of Liquid Mixtures. Annu. Rev. Chem. Biomol. Eng. 2010, 1, 101-122.

(39) Nti-Gyabaah, J.; Gbewonyo, K.; Chiew, Y. C. Solubility of Artemisinin in Different Single and Binary Solvent Mixtures between (284.15 and 323.15) K and NRTL Interaction Parameters. J. Chem. Eng. Data 2010, 55 (9), 3356-3363.

(40) Horosanskaia, E.; Seidel-Morgenstern, A.; Lorenz, H. Investigation of Drug Polymorphism: Case of Artemisinin. Thermochim. Acta 2014, 578, 74-81.

(41) Chan, K. L.; Yuen, K. H.; Takayanagi, H.; Janadasa, S.; Peh, K. K. Polymorphism of Artemisinin from Artemisia Annua. Phytochemistry 1997, 46, 1209-1214. 
(42) Mullins, E.; Liu, Y.; Ghaderi, A.; Fast, S. D. Sigma Profile Database for Predicting Solid Solubility in Pure and Mixed Solvent Mixtures for Organic Pharmacological Compounds with COSMOBased Thermodynamic Methods. Ind. Eng. Chem. Res. 2008, 47 (5), 1707-1725.

(43) Schröder, B.; Santos, L. M.; Rocha, M. A.; Oliveira, M. B.; Marrucho, I. M.; Coutinho, J. A. Prediction of Environmental Parameters of Polycyclic Aromatic Hydrocarbons with COSMO-RS. Chemosphere 2010, 79 (8), 821-829.

(44) Kong, W.; Sun, R.; Gao, Y.; Nan, G.; Yang, G.; Li, Y. Dissociation Constants and Solubilities of Dalbergin and Nordalbergin in Different Solvents. J. Chem. Eng. Data 2015, 60 (9), 2585-2593.

(45) Klamt, A. COSMO-RS: From Quantum Chemistry to Fluid Phasethermodynamics and Drug Design; Elsevier: Amsterdam, 2005.

(46) Do, H.; Wheatley, R. J.; Hirst, J. D. Microscopic Structure of Liquid 1,1,1,2-Tetrafluoroethane (R134a) from Monte Carlo Simulation. Phys. Chem. Chem. Phys. 2010, 12 (40), 13266-13272.

(47) Suberu, J. O.; Yamin, P.; Leonhard, K.; Song, L.; Chemat, S.; Sullivan, N.; Barker, G.; Lapkin, A. A. The Effect of O-Methylated Flavonoids and Other Co-Metabolites on the Crystallization and Purification of Artemisinin. J. Biotechnol. 2014, 171, 25-33.

(48) Chemat, F.; Vian, M. A.; Cravotto, G. Green Extraction of Natural Products: Concept and Principles. Int. J. Mol. Sci. 2012, 13 (7), $8615-8627$.

(49) Rombaut, N.; Tixier, A. S.; Bily, A.; Chemat, F. Green Extraction Processes of Natural Products as Tools for Biorefinery. Biofuels, Bioprod. Biorefin. 2014, 8 (4), 530-544. 